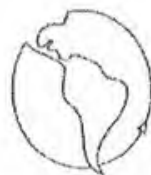

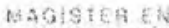
ANTROPOLOGIA Y DESAAFOLLO

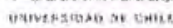

\title{
Signos de la Exclusión: Las Relaciones de Género y el
} Juego de lo Invisible/Visible

\section{Sonia Montecino Aguirre smonteci@uchile.d}

Nos abocaremos, en este breve ensayo, a reflexionar, a partir de una paradoja que ilustra la tensión inclusión/exclusión social y que, a la vez, nos permite aproximarnos a la posibilidad de la igualdad en la diferencia como resolución, teórica y política, a la misma. Se trata de una paradoja situada: en Chile una mujer ostenta el máximo poder político de la República -la presidencia-, sin embargo, se mantiene una débil participación laboral femenina', una desigual remuneración, un bajo acceso a cargos de decisión (en las distintas esferas políticas, empresariales, universitarias), y un aumento y cambio en el tipo de violencia contra las mujeres: el femicidio ${ }^{2}$, entre otras iniquidades.

Se puede concluir de manera fácil que la explicación a esta paradoja radica en que nada indica que el acceso al máximo poder político por parte de una mujer posea como correlato una sociedad donde su inclusión, económica, social y simbólica esté garantizada. La antropología del género, en ese sentido, ha demostrado que hay numerosas comunidades humanas donde las mujeres pueden tener dominio sobre ciertas esferas, pero no poder y prestigio en todas las que conforman la vida social (Ortner y Whitehead, 1981). Si lo anterior es cierto, lo que nos interesa mostrar con esta paradoja situada son las formas con que operan, en el caso concreto de las relaciones de género en Chile, ciertos mecanismos contradictorios de inclusión y exclusión social.

\footnotetext{
Las úlcimas cifras oficiales plantean una participación del 37,5\% a diferencia del $45 \%$ de otros países de América Latina (Entrevista a Ministra del Sernam, El Mercurio, 14 de agosto, 2006).

2 Las cifras que manejan Ongs dedicadas a estos temas o centros académicos como la FLACSO, coinciden en que se están produciendo como promedio alrededor de 70 femicidios antales en Chile. La revista Ercilla (5-18 de diciembre del 2005) cita el trabajo de Luis Aravena quien contabilizó los femicidios aparecidos en el Diario La Cuarta entre 1990 al 2000, Ilegando a 581 casos, de los cuales 345 fueron perpetrados por hombres que habian mantenido alguna relación íntima con la mujer.
} 
Entendemos que los conceptos de inclusión y exclusión emergen con el cambio de una concepción "...en la que todos los miembros de la sociedad estaban ligados por medio de un contrato social a un Estado de Bienestar, (mientras que hoy) la sociedad excluye cada vez a más personas. De hecho se da un cambio de tendencia que Niklas Luhmann ha descrito como la transformación de una sociedad de inclusión a una sociedad de exclusión" (Kurnitzky, 2002: 56). Si antes se hablaba de marginalidad e integración al desarrollo dentro de una economía capitalista, hoy día esos términos no expresan las nuevas formas en que las personas y los grupos se ven vulnerados, muchas veces, en sus derechos humanos mínimos o en sus derechos culturales. Pero, de manera muy evidente -y en eso hay consenso por parte de los especialistas-, el proceso de globalización es el que ha traído consigo la dinámica a gran escala de la exclusión social ${ }^{3}$.

La lucha de las mujeres por alcanzar niveles cada vez más amplios de "inclusión" dentro de la sociedad son antiguos y han formado parte de una gran revolución, de un profundo cambio vivido a lo largo del siglo XX que ha pasado por demandas en el plano de lo público: derecho al trabajo, a la educación, a la participación política -entre otras- $y$ en el de lo privado: al control de su propio cuerpo, a la anticoncepción, a la valorización del trabajo doméstico y reproductivo.

Un balance de esas demandas da cuenta que una vez alcanzados ciertos derechos, las mujeres han debido plantear otros, incluso aun cuando los anteriores no han sido logrados a plenitud. Por otro lado, la reflexión teóri-

\footnotetext{
Manucl Antonio Garretón (1999) ve cuatro transformaciones producidas por la globalización: la primera, se relaciona con la homogeneización mediática de la cultura de masas y la impugnación de ésta por lo que llama la "explosión de las identidades" comunitaristas; la segunda, con la resignificación de los Estados nacionales a través de nuevas formas de integración y democratización, pero tambièn de la creación de bloques o espacios supranacionales, mas sin formas de gobierno globalizado; la tercera, “...es la expulsión de vastos sectores de la humanidad y la generación, por parte de cada nuevo mecanismo de globalización, de nuevas formas de exclusión. La masa de excluidos y marginales, que puede alcanzar a más de la mitad de la humanidad... a diferencia de formas conocidas de exclusión vinculadas a la dominación y explotación, tiene relaciones muy débiles con la sociedad, y se vincula, pasivamente, a la globalización en forma puramente simbólica o mediática" (3) y, por último, la cuarta, es una globalización doninada por grandes potencias, o poderes económicos, pero con una contracara que Garretón llama "la mundialización de la sociedad civil", expresada en las asambleas internacionales sobre medio ambiente, pobreza, mujer, derechos humanos, etc.
} 
ca ligada al feminismo y a los estudios de la mujer o de género han producido conocimientos críticos de enorme relevancia, así como develamientos de ciertas estructuras que operan en la constitución de lo social que han sido invisibilizadas por muchos de los modelos teóricos de las ciencias sociales, como por ejemplo, el que las diferencias sexuales, simbolizadas y socializadas a través de los sistemas de género construyen, en la base de todas las culturas, las relaciones de poder (de igualdad, complementación o desigualdad). Si lo social se ha construido a partir de un sacrificio (de lo natural) $)^{4}$ ello supone que "La civilización se desprende de la tensión de los sexos que es fundamento y punto de arranque del proceso civilizador. Y la relación mutua entre los sexos determina, en última instancia, la totalidad de las relaciones de violencia en la sociedad. Es el medidor en la escala de la civilización. En la historia del desarrollo del culto sacrificial es posible identificar el siguiente proceso: desde la víctima real del sacrificio, a través de las prácticas simbólicas, hasta el contrato social" (Kurnitzky, 2002:64).

Las consecuencias de esta dimensión cultural (simbolización de los cuerpos en categorías femeninas y masculinas) de la sociedad humana trajo consigo una discursividad sobre las diferencias sexuales (lo biológico), constituidas como diferencias de género, que casi siempre entrañó (y entrañan) una jerarquización y dominio, un sistema de valoración social. Como sostiene Segato - discutiendo algunas narrativas antropológicas que plantean desmontar, recurriendo a algunos mitos, la noción de subordinación universal de la mujer- el papel de instituir la ley es masculino y “....permanece fuertemente asociado con la virilidad ideológica y trascendente del dios. La autoridad, por tanto, no es neutra, no está encarnada en un figura andrógina, sino radicada en una divinidad que exhibe los atributos de la masculinidad. De este modo, esa figura, esa posición en el discurso constituye... la llave de la comprensión de lo que es la masculinidad... Se trata, una vez más, de la ley fálica de la interdicción, de la separación, del límite y del orden" (2003:71).

De estas consideraciones se desprende que una de las preocupaciones centrales en las reflexiones sobre género ha sido el concepto de igualdad y

\footnotetext{
4 Numerosas vertientes del pensamiento sostienen esta idea, desde Freud, Lévi Strauss, Giraard, siendo Kurnitzky quien plantea la hipótesis que este "sacrificio" fue en sus comienzos femenino.
} 
sus horizontes posibles de alcanzar. La búsqueda de igualdad entre hombres y mujeres, para lograr la justa inclusión de éstas a todas las esferas, supuso en las sociedades modernas una cierta identidad de las mujeres (una universalidad de la sujeción y dominación); sin embargo, la incorporación, por ejemplo, al universo del trabajo remunerado puso de manifiesto que de acuerdo a la pertenencia de clase, la condición de las mujeres era distinta, como también lo era su posición dentro de las estructuras. Lo mismo sucede cuando la mirada se posa en las características étnicas y de generación, entre otras. Por eso, la "identidad" mujer cedió su paso a un concepto plural y cambiante del sujeto femenino, un sujeto cuya posición se desplaza de acuerdo a los contextos en los cuales se realiza su existencia.

Sin embargo, la lucha por la igualdad con los hombres siguió siendo una demanda, en la medida en que en las diversas prácticas sociales, con grados siempre crecientes de inclusión, las mujeres -o la categoría social de las mujeres más precisamente- se ubican indefectiblemente en los lugares más desvalorizados en el plano salarial, del poder, de la toma de decisiones, de los espacios de prestigio, del respeto social. Por ello, la utopía de la igualdad y de la inclusión total de lo femenino -como correlato simbólico del discurso sobre el cuerpo de las mujeres- propuso nuevas formas de reflexión y acción política. La igualdad -así quedó claro, sobre todo para las intelectuales europeas- no consistía en ocupar las mismas profesiones u oficios de los hombres, ni siquiera en conquistar poder dentro de la política, en la medida en que estos lugares públicos -inmersos en espacios de desigualdad económica- operan bajo la lógica de la competencia, la dominación y de manera más compleja y potente, suponen un imaginario que excluye, culturalmente, a lo femenino en tanto sujeto de relaciones igualitarias.

De esa constatación emerge la postura de lo femenino como diferencia. Pero, de una diferencia que al constituirse en desigualdad provoca la marginación, la exclusión y, al mismo tiempo, porta la invisibilidad de los mecanismos que llevan a su acantonamiento en lo precario y lo devaluado. Por eso, igualdad y diferencia promovieron un campo de tensión en medio de nuevas luchas emergidas de las cambiantes condiciones de las mujeres. La inclusión en el mercado de trabajo tuvo que traer consigo códigos relaciona- 
dos al abuso sexual, a la maternidad, a la discriminación, y recientemente a la lucha por igualdad de salarios 5 . Lo que se desprende de ello es que la "diferencia" portada por lo femenino se estrella con la supuesta "igualdad" que implica acceder a la arena de un espacio dominado históricamente por lo masculino $0^{6}$. Lo real es que la diferencia femenina puesta en acción en el universo de lo público conlleva desigualdad. No es extraño, entonces, que los Estados promuevan políticas de paridad, de cuotas, de discriminación positiva, impulsados básicamente por las instituciones internacionales de promoción o defensa de los derechos humanos y de las mujeres?

No obstante, las diversas políticas para lograr la igualdad entre hombres y mujeres no contemplan una reforma en el plano más álgido de constitución de lo social: el mundo de lo íntimo, de lo privado, de la reproducción de lo doméstico (de la desigualdad en la división sexual del trabajo doméstico) y de las relaciones de parentesco (cuyo corolario es una cierta organización de los afectos). Es decir, allí donde las relaciones entre hombres y mujeres producen la vida humana y hacen posible el decurso de lo filogenético. Pero sobre todo, no se produce reflexión crítica respecto a la tensión que sobre lo doméstico genera lo público en cuanto a la valoración social "generizada" de las prácticas que lo constituyen y a su inclusión como elemento constitutivo de la vida social.

5 Todo ello impulsado por los movimientos feministas de la sociedad civil globalizada, como sostiene Garretón, quienes han construido estándares de medición mundial para resguardar los derechos de las mujeres, así como diversas asambleas $y$ cumbres internacionales que permiten conocer el grado de avance cn esas materias de los distintosEestados.

6. No se ha generado todavía una discusión sobre el papel de las mujeres en otro espacio de dominio masculino: el mundo del pensamiento y de la academia. Más allá de la constatación de su enorme marginación, no tanto en el acceso al espacio académico sino en la representación de lo femenino dentro de las teorias e interpretaciones sobre lo social. La exclusión en ese plano es enorme, y llama la atención que son en su mayoría mujeres las que comienzan a realizar la labor de inclusión de lo femenino en las respectivas disciplinas; pero también es conocida la invisibilización de sus aportes. Justamente esta misma reflexión sobre igualdad y diferencia producida a funes de los 70 por las feministas, recién comienza a cobrar un significado cuando autores hombres lo "elevan" a discusión general.

1. Bihr y Pfefferkorn (2002) plantean una diferencia entre la "igualdad formal" y la "igualdad real": "Bien que l'égalité entre les sexes soit désormais un principe hautement affirmé au sein de l'espace public, et en dépit des changements importants intervenus en l'es pace d'une génération, les repports hommes/femmes restent globalement des rapports inégalitaires. L'égalité formelle ainsi proclammé masque malles inégalités récles qui persistent" (352). 
A esta tensión entre público/privado se agrega una mayor, que de algún modo la contiene: la del contrato y el estatus. Segato piensa que estos son dos ejes interconectados, uno vertical, vinculado a relaciones de entrega y expropiación, y uno horizontal ligado a las relaciones de alianza y competencia. Ambos conforman un "sistema único", de equilibrio inestable. El horizontal se organiza ideológicamente en la noción de "contrato entre iguales", y el vertical se articula en torno al "...mundo premoderno de estamentos y castas. En ambos ejes, los miembros son portadores de índices diacríticos de su posición relativa" (2003:253). De este modo, aunque el contrato y el estatus son universos de sentido que se originan en tiempos diferentes, coexisten. Nosotras pensamos que precisamente la larga duración del sistema de estatus es lo que, en nuestras sociedades latinoamericanas y especialmente en Chile, permite dar cuenta de las contradicciones entre las prácticas sociales ligadas a las relaciones de género (ancladas al orden del estatus) y las políticas de igualdad (afincadas en el orden del contrato).

El campo de fuerzas entre el sistema del estatus y el del contrato adquiere su máxima potencia cuando las mujeres descalzan al primero transitando y desplazándose de lo privado a lo público. La socialización en determinadas concepciones de género se producen en una imbricación entre lo íntimo y lo colectivo, en el aprendizaje de la lengua y sus sentidos, en la memoria de modelos femeninos y masculinos que se transmite oral y gestualmente $y$ que en cada clase tendrá su realización particular. Los códigos de género así transmitidos son los del estatus: las jerarquías entre hombres y mujeres, el control del cuerpo femenino, la violencia sobre ese cuerpo cuando no acata el poder masculino, el valor de la maternidad, el trabajo reproductivo y doméstico como una labor de mujeres, etc., todos ellos construyendo un sistema de valores y posiciones, así como un imaginario que, como sabemos, tiene por función "naturalizar" los argumentos de las desigualdades que produce.

La contradicción y el conflicto emergen cuando los sujetos femeninos y masculinos se "encuentran" en el espacio del contrato, de la "igualdad formal" portando todos aquellos signos que los han construido como categorías dentro de una jerarquía de mayor $y$ menor prestigio, de mayor y menor autonomía. No es extraño entonces que se deban crear, entre otras medi- 
das, códigos de buenas prácticas laborales, que tienden a paliar -desde la escritura y la "ley"- las iniquidades que el sistema del estatus y su imaginario producen cuando las mujeres "usurpan" los espacios considerados por éste como propios y que otorgan prestigio masculino. Para autoras como Segato y Pateman (1993) las nuevas formas de violencia contra las mujeres se explican por esta dinámica del estatus y el contrato.

Podemos desprender entonces que la inclusión de las mujeres a las distintas esferas sociales de poder tiene su muro de contención en la mantención de una cultura basada en el orden del estatus y podemos colegir también que la superación de esta valla no es simplemente la promulgación de leyes de incorporación a las estructuras económicas y políticas. Por cierto, y esto es importante de consignar, esa cultura del estatus no sólo opera en el caso de las mujeres y los hombres, sino también y de manera simultánea en relación a la clase social, a la etnia, a la generación, todas ellas posiciones y condiciones sociales que forman parte de los sujetos y de su género.

Por ello se torna necesario problematizar el vínculo entre igualdad y diferencia. El dilema, en el caso de las mujeres se traduce en una doble encrucijada: la igualdad, en términos culturales, supondría diluir su especificidad y "ser como" los hombres. La política de la diferencia, por el contrario, plantea ser iguales, pero "como mujeres". Ese "ser como las mujeres" en muchos casos se anida en la construcción de lo femenino como maternidad (el cuerpo diferente). Pero, lo anterior nos conduce al predominio del sistema del estatus, que es el que precisamente anuda la desigualdad. La construcción de una "nueva ciudadanía" y de procesos crecientes de inclusión para las mujeres tendría necesariamente que pasar por una transformación del sistema del estatus y por una nueva concepción de la oposición público/ privado en la cual, como dice Chantal Mouffe (1993): “..cada situación sea un encuentro entre lo privado y lo público, puesto que cada empresa es privada aunque nunca sea inmune a las condiciones públicas prescritas por los principios de la ciudadanía" (16).

Esa transformación debería traer consigo también formas nuevas de valoración social a esos espacios, pues mientras se siga concibiendo un imaginario simbólico que coloca a lo doméstico indefectiblemente ligado a lo 
femenino ${ }^{8}$, la igualdad entre hombres y mujeres en el plano de lo público no será garantía de una plena inclusión, pues el cruce de los sujetos entre el orden del trabajo -remunerado-y de lo público -la política-se engarzan, lo queramos o no, con el de la reproducción doméstica cotidiana -la política de los afectos-. Por ello también, la inclusión social no pasa sólo por acciones en el ámbito económico y político, sino por el simbólico, por el poder de la interpretación, por el cambio en los patrones de construcción de los proyectos colectivos, pues es allí donde se juega de manera prístina la visibilidad o invisibilidad de la exclusión. En nuestro caso, si no somos capaces de desmontar el sistema del estatus -que como sabemos opera con antiguas y nuevas formas de elaboración de las nociones de prestigio social-difícilmente podremos avanzar en crecientes espacios de igualdad.

La reflexión sobre la igualdad en la diferencia que han colocado desde hace varias décadas las intelectuales y pensadoras de los estudios de género, es una que sin duda toca a temas globales como el valor de la diversidad versus el valor de lo universal, el respeto a lo distinto versus el respeto a los derechos colectivos que hoy día es uno de los nudos en los cuales puede lecrse la trama que afecta a la sociedad en su conjunto. En el caso específico de la diferencia ligada a los géneros, el urdido roza a los cimientos en los cuales se estructura y reproduce la vida social, a la distribución del poder y a la organización de los afectos.

A partir de estas consideraciones la paradoja con la cual abrimos nuestro trabajo puede ser comprensible y hace visible la dinámica entre el sistema del estatus y el del contrato que supone inclusiones "defectuosas" o desiguales de las mujeres en el plano laboral y político. Tenemos la hipótesis que en la medida en que no se resuelva y deconstruya críticamente el primero se mantendrá la exclusión femenina en muchos niveles, más aun, que se producirá un aumento de violencia contra las mujeres -es interesante seńalar que en el caso de algunos países nórdicos, donde existe paridad en muchas esferas so-

*. Es interesante señalar que una encuesta recientemente efectuada por el diario La Tercera pone de manifiesto que tanto hombres como mujeres chilenos (as) consideran en casi un $77 \%$ que son las mujeres quienes mejor desempeńan las actividades reproductivas, de la casa; así como las administrativas y de mancjo del presupuesto. 
ciales, o en el de Espańa (exportador de los modelos de igualdad de oportunidades a los países latinoamericanos), la violencia de todo tipo ha crecido de manera alarmante-. Ello no es extraño pues, si seguimos a Kurnitzky: el sacrificio femenino forma parte de la constitución de la cultura, está arraigado en la psiquis y se ha rearticulado -desde nuestra óptica- en una sociedad donde las mujeres han subvertido ese orden fundante exigiendo no sólo ser dadoras de la vida, sino ser dadoras de sentido, como dice Julia Kristeva. Por ello, retornarlas a su "cauce" por medio de la violencia es el corolario de un proceso que no sólo afecta a las relaciones de género, sino a las relaciones entre culturas, generaciones, clases, etc.

De ahí deriva nuestra insistencia en que la igualdad como necesario horizonte utópico que produce mayores niveles de inclusión social, en el caso que nos ocupa, supone una nueva manera de concebir la posición y condición de hombres y mujeres y su desplazamiento intercambiable y constante entre lo público y lo privado como esferas también con igual valoración y prestigio simbólico. Asimismo, implica un constante desmontaje de los signos que nos impelen con sus atavismos a reproducir gestos que anclados en el imaginario social producen exclusión y violencia. Una reforma en los afectos, quizás nos pueda ayudar también a alcanzar esos nuevos senderos ciudadanos en medio de una sociedad que ha necesitado siempre de ellos para subsistir.

\section{Bibliografia}

Bihr, Alain y Pfefferkorn, Roland (2002). Hommes/Femmes: quelle égalité, Paris Les Editions de l'Atelier.

Garretón, Manuel Antonio (1999). América Latina: un espacio cultural en el mundo globalizado, Bogotá, Convenio Andrés Bello.

Mouffe, Chantal (1993). "Feminismo, ciudadanía y política democrática radical” en Debate Feminista, № 7, 1993, México.

Ortner, Sherry y Whitehead (1981). "Accounting for sexual meanings", en Sexual Meanings. The cultural construction of Gender and Sexuality, Cambridge, Cambridge University Press. 
Pateman, Carole (1995). El contrato sexual, Barcelona México, Anthropos/ UNAM.

KURNITZKY, Horst (2002). Una civilización incivilizada. El imperio de la violencia en el mundo globalizado, México, Editorial Océano.

Segato, Rita Laura (2003). Las estructuras elementales de la violencia. Ensayos sobre género entre la antropología, el psicoanálisis y los derechos humanos. Buenos Aires, Prometeo/3010 Universidad Nacional de Quilmes. 